\title{
Fibro-odontoma ameloblástico. Reporte de caso clínico y revisión de la literatura
}

\author{
Ameloblastic fibrodontoma clinical case \\ report and literature review
}

\author{
José Eduardo Cruz López,* José Ernesto Miranda Villasana, ${ }^{\ddagger}$ María Luisa López Salgado, ${ }^{\S}$ \\ Nicolás Santiago Álvarez," Dailyn Rodríguez Rodríguez ${ }^{\mathbb{I}}$
}

\section{RESUMEN}

El fibro-odontoma ameloblástico es un tumor odontogénico benigno extremadamente raro en frecuencia, asociado a pacientes jóvenes sin predilección por género. Clásicamente se considera una tumoración odontogénica epitelial que contiene ectomesénquima con la formación de tejido duro dentario; sin embargo, en la última actualización de la clasificación de tumores odontogénicos de la Organización Mundial de la Salud (OMS) del año 2017, se considera una variante inusual del fibroma ameloblástico. Presentamos el caso clínico de un paciente femenino de nueve años con diagnóstico histopatológico definitivo de fibro-odontoma ameloblástico, con su manejo quirúrgico, así como una revisión sistemática de la literatura.

Palabras clave: Fibro-odontoma ameloblástico, fibroma ameloblástico.

\section{ABSTRACT}

Ameloblastic fibro-odontoma is an extremely rare benign odontogenic tumor in frequency associated with young patients without gender predilection, it is classically considered an epithelial odontogenic tumor that contains ectomesenchyme with the formation of hard dental tissue, however, in the last update of the classification of odontogenic tumors of the World Health Organization (WHO) of 2017, considers it an unusual variant of ameloblastic fibroma. We present the clinical case of a nine-year-old female patient with a definitive histopathological diagnosis of ameloblastic fibroodontoma, with its surgical management, as well as a systematic review of the literature.

Keywords: Ameloblastic fibro-odontoma, ameloblastic fibroma.

* Residente de cuarto año de la Especialidad de Cirugía Oral y Maxilofacial. Hospital Regional «Gral. I Zaragoza», ISSSTE.

‡ Jefe del Servicio de Cirugía Oral y Maxilofacial. Hospital Regional «Gral. I Zaragoza», ISSSTE.

$\S$ Jefe del Servicio de Cirugía Maxilofacial del Hospital para el Niño, IMIEM, Toluca, Estado de México.

" Médico adscrito del Servicio de Cirugía Maxilofacial del Hospital para el Niño, IMIEM, Toluca, Estado de México.

" Residente de primer año de la Especialidad de Cirugía Oral y Maxilofacial. Hospital Regional "Gral. I Zaragoza», ISSSTE.

Correspondencia:

José Eduardo Cruz López

Hospital para el Niño, IMIEM, Toluca, Estado de México.

Paseo Cristóbal Colón S/N,

Isidro Fabela Primera Sección, 50170,

Toluca de Lerdo, México.

Tel: 7222174044

E-mail: lalo_cruz89@ hotmail.com 


\section{INTRODUCCIÓN}

El fibro-odontoma ameloblástico (FOA) fue descrito por primera vez por Hooker en el año de 1967, distinguiéndolo como uno de los odontomas ameloblásticos más agresivos. Es un tumor odontogénico benigno extremadamente raro en frecuencia, clásicamente figura como una tumoración odontogénica epitelial que contiene ectomesénquima con formación de tejido duro dentario; sin embargo, en la última actualización de la clasificación de tumores odontogénicos de la Organización Mundial de la Salud (OMS) del año 2017 , se considera una variante inusual del fibroma ameloblástico ( $F A)$, al igual que el fibrodentinoma ameloblástico (FDA), debido a que presenta características histológicas similares asociadas al desarrollo de tejidos duros odontogénicos (Tabla 1). 1-3

Representa tan sólo del 1 al $3 \%$ de todos los tumores odontogénicos y comúnmente es encontrado en radiografías de rutina en pacientes con ausencia de erupción dental o por presentar aumentos de volumen asintomáticos. Afecta principalmente a pacientes jóvenes entre la primera y segunda década de la vida, no muestra predilección por género, aunque algunos autores sostienen una leve tendencia hacia el género masculino. ${ }^{2,4}$

Clínicamente se presenta con mayor frecuencia en la región mandibular posterior y se caracteriza por ser una lesión asintomática, de tamaño variable y crecimiento lento que genera expansión ósea. La mucosa que lo recubre es normal y presenta escasa tendencia a la infección y usualmente se encuentra asociado a dientes no erupcionados. ${ }^{2,5}$

En la exploración radiográfica se puede observar un patrón radiolúcido expansivo, que generalmente contiene focos radiopacos pequeños, ya sean solitarios o múltiples de tamaño irregular y a menudo asociado a dientes impactados. Varía de pocos milímetros a centímetros generando expansión de tablas óseas y desplazamiento de estructuras anatómicas. La patogenia del FOA parece deberse a una proliferación anormal del epitelio odontogénico de un germen dental permanente, que ejerce un efecto organizador sobre el elemento mesodérmico con la formación de tejido dental calcificado. 2,6,7

Histológicamente, el FOA se ha clasificado como un fibroma u odontoma ameloblástico. Se caracteriza por presentar islotes y cordones de epitelio odontogénico inmerso en un tejido conectivo embrionario que imita la pulpa dental primitiva. La formación de osteodentina y esmalte también puede observarse microscópicamente, el tejido conectivo es moderadamente celular, con fibroblastos en forma de huso., ${ }^{2,7,8}$

\section{CASO CLÍNICO}

Presentamos el caso clínico de un paciente femenino de nueve años, el cual acude al Servicio de Cirugía Maxilofacial del Hospital para el Niño, IMIEM, Toluca, por presentar aumento de volumen localizado en región posterior mandibular de lado izquierdo con un mes de evolución, asociado a sintomatología dolorosa. A la exploración maxilofacial se observa asimetría facial a expensas de aumento de volumen en tercio inferior facial de lado izquierdo (Figura 1), con piel que lo recubre de características normales, firme y dolorosa a la digitopresión. Intraoralmente se corresponde con un aumento de volumen en fondo de vestíbulo y reborde alveolar de área molar inferior izquierda con dimensión aproximada de 4 $\mathrm{cm}$ de diámetro, no desplazable a la manipulación, con mucosa superficial ulcerada secundario a traumatismo oclusal y zonas blanquecinas de apariencia calcificada en el centro de la lesión (Figura 2).

Radiográficamente cuenta con ortopantomografía donde se observa una imagen de patrón mixto a expensas de una zona radiolúcida unilocular bien delimitada con radiopacidades en su interior. Dicha imagen abarca la totalidad del cuerpo, parte del ángulo y rama mandibular del lado izquierdo; desplazando conducto dentario inferior, así como órganos dentarios adyacentes, sin producir rizólisis; otro dato importante es la agenesia de segundo y tercer molar (Figura 3).

Para complementar la valoración imagenológica, se solicita tomografía computarizada de haz cónico donde se observa, además de lo antes mencionado, expansión y adelgazamiento de ambas corticales, respetando el borde basal mandibular (Figura 4).

Debido a estos hallazgos se continúa protocolo quirúrgico, iniciando con biopsia insicional, obteniéndose como resultado preliminar fibroma ameloblástico, por lo que se decide realizar enucleación y curetaje de la lesión con extracción del órgano

Tabla 1: Clasificación de tumores odontogénicos de la Organización Mundial de la Salud, año 2017.

Tumores odontogénicos epiteliales mixtos y mesenquimatosos benignos

Fibroma ameloblástico

\begin{tabular}{cc}
\hline & Variantes \\
$\begin{array}{c}\text { Fibro-odontoma } \\
\text { ameloblástico }\end{array}$ & $\begin{array}{c}\text { Fibro-dentinoma } \\
\text { ameloblástico }\end{array}$ \\
\hline
\end{tabular}




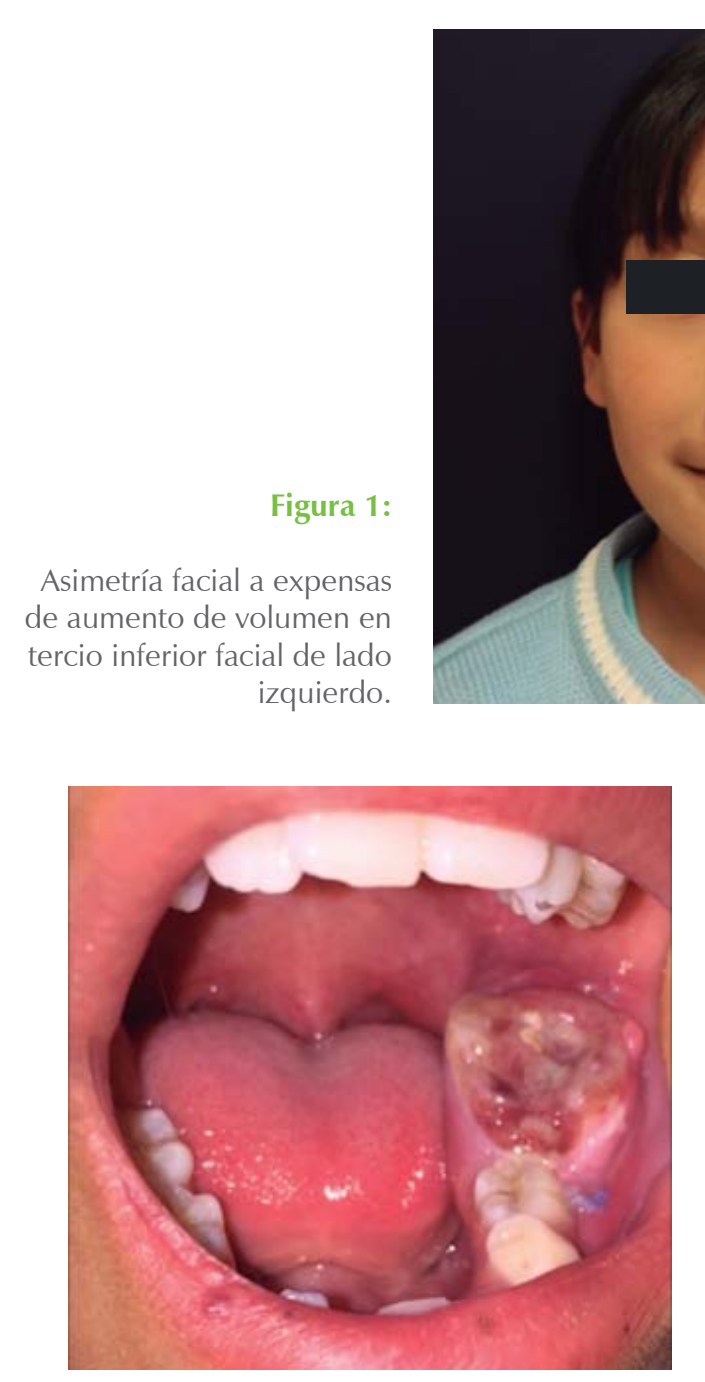

Figura 2: Exploración intraoral. Aumento de volumen en fondo de vestíbulo y reborde alveolar de área molar inferior izquierda con dimensión aproximada de $4 \mathrm{~cm}$ de diámetro, mucosa superficial ulcerada secundario a traumatismo oclusal y zonas blanquecinas de apariencia calcificada en el centro de la lesión.

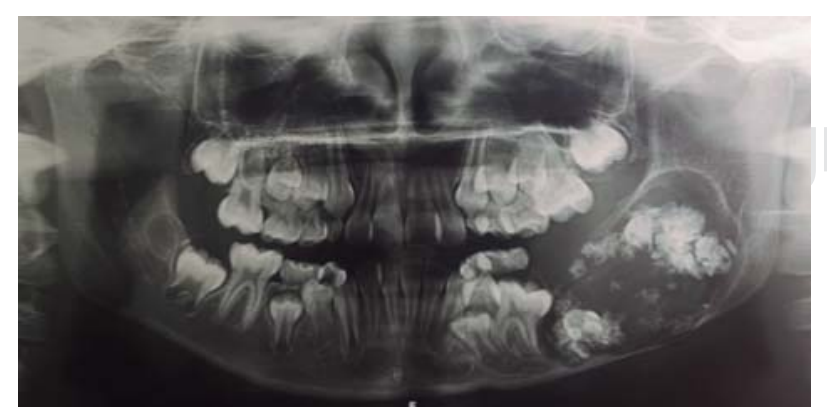

Figura 3: Se observa una imagen de patrón mixto a expensas de una zona radiolúcida unilocular bien delimitada con radiopacidades en su interior. dentario retenido, así como colocación de tornillos de fijación intermaxilar con fines preventivos ante posible riesgo de fractura patológica. Se obtuvo una pieza quirúrgica de tejido blando de forma aproximadamente ovoidal de superficie lisa con zonas rugosas, con dimensiones de $45 \times 38 \times 25 \mathrm{~mm}$, la cual se envía a estudio histopatológico definitivo (Figura 5).

El acto quirúrgico se realizó de manera conservadora, logrando adecuada síntesis del abordaje sin tensión. Debido a que se consiguió respetar la integridad de las corticales óseas, el borde basal mandibular y el periostio se descartó la necesidad de injerto óseo o material de reconstrucción. Durante el estudio histopatológico, se identifica una lesión de estirpe odontogénica formada por múltiples lóbulos de tejido de aspecto mesenquimatoso inmaduro hipercelular, en el que se observan abundantes islas, cordones y yemas de epitelio de tipo ameloblástico con capa basal cuboidal a columnar y pequeños grupos de células que recuerdan al retículo estrellado del órgano del esmalte en su porción central, las cuales están dispersas en forma desorganizada en toda la lesión y en algunas áreas adyacentes a zonas de formación de tejidos dentales mineralizados (dentinoide y esmalte). La lesión se encuentra bien delimitada por tejido fibroso laxo maduro bien vascularizado compatible a fibro-odontoma ameloblástico.

\section{DISCUSIÓN}

EI FOA se plasma como una entidad neoplásica odontogénica benigna agresiva, debido a su crecimiento expansivo e indoloro, generalmente descu- 

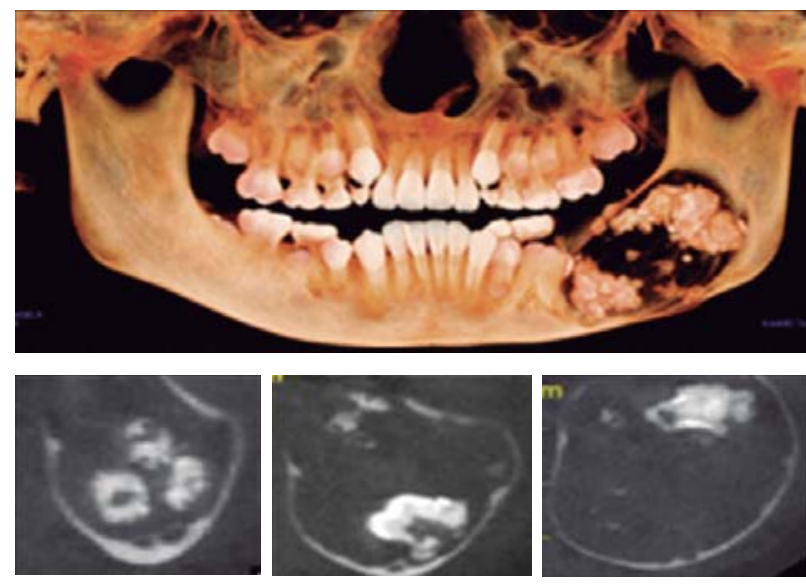

Figura 4: En TC de haz cónico se observa expansión y adelgazamiento de ambas corticales, respetando el borde basal mandibular.

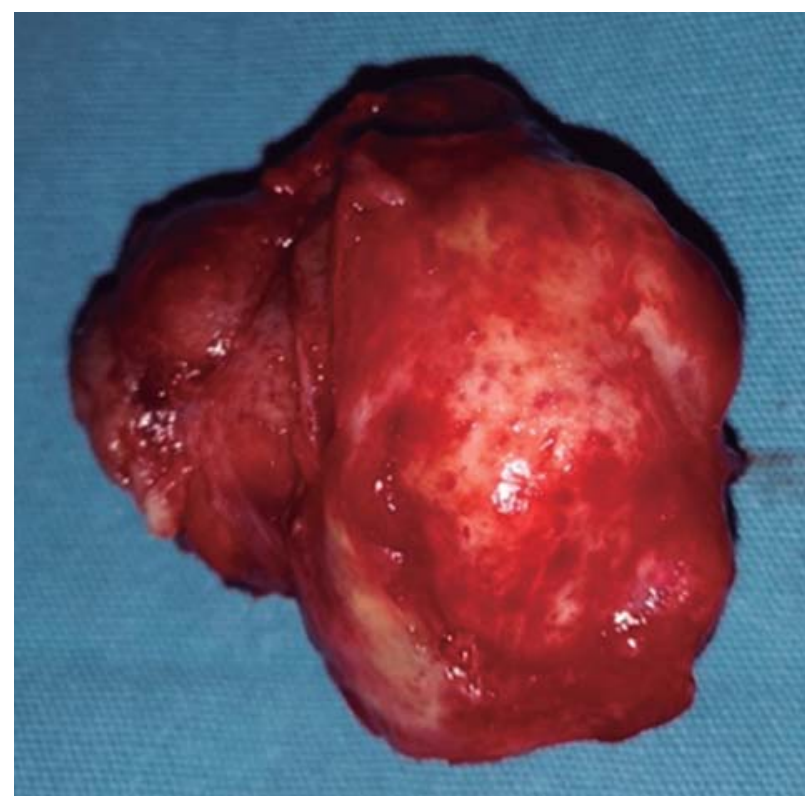

Figura 5: Pieza quirúrgica de tejido blando de forma aproximadamente ovoide de superficie lisa con zonas rugosas, con dimensiones de $45 \times 38 \times 25 \mathrm{~mm}$.

bierto por hallazgo radiográfico, siendo el principal motivo de consulta un aumento de volumen y retraso en la erupción dentaria. ${ }^{1}$

Autores como Slootweg no ven diferencia en frecuencia de aparición en maxilar o mandíbula; sin embargo, algunos otros como Boxberger en su metaanálisis lo considera más frecuente en la región posterior mandibular, con una proporción de 1.85:1 respectivamente..$^{4,5}$
Actualmente se sabe que el FOA se trata de una variante inusual del fibroma ameloblástico, pero asociado al desarrollo de tejidos duros odontogénicos, descartando la teoría de Cahn \& Blum quienes postulaban que el FOA, FDA y FA se trataban de diferentes estados de desarrollo de una misma entidad que finalmente maduraba en un odontoma. ${ }^{3,4,8}$

Por lo anterior, debemos tomar en cuenta al odontoma complejo inmaduro como diagnóstico diferencial, así como al tumor odontogénico epitelial calcificante, más frecuente en mandíbula en zona de premolares; o el tumor odontogénico adenomatoide, el cual es extremadamente raro y más común en región anterior mandibular. ${ }^{5}$

Su pronóstico es excelente, sin embargo, se ha descrito su recurrencia en distintos periodos luego de su enucleación con o sin conservación de sus dientes asociados, incluso autores como Herzog reportan la existencia de recurrencia luego de 12 años de seguimiento postquirúrgico con transformación maligna a fibrosarcoma ameloblástico y metástasis. ${ }^{2}$

La planificación del tratamiento se verá directamente relacionada con el diagnóstico histopatológico específico, el tamaño de la lesión, la edad del paciente y el comportamiento biológico de la lesión, pudiendo variar desde una pequeña enucleación con curetaje del lecho quirúrgico hasta una resección extensa. ${ }^{2,5}$

El tratamiento de elección es la enucleación o escisión local conservadora con extracción de dientes retenidos asociados, para otorgar un excelente pronóstico y mínima recurrencia. Su potencial de transformación por sí solo no justifica un tratamiento radical. Algunos autores utilizan injertos autógenos o materiales aloplásticos para preservar el volumen óseo perdido. Sin embargo,

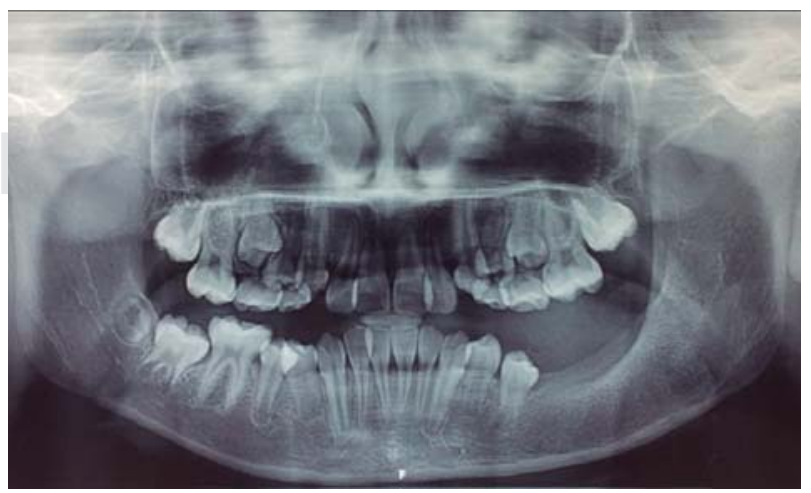

Figura 6: Control radiográfico a los seis meses. 
si durante la enucleación se preserva el periostio y las corticales óseas, esto permite una regeneración espontánea en defectos de pacientes jóvenes (Figura 6).2,5,7,8

\section{CONCLUSIONES}

La localización del FOA, edad y género de nuestro paciente coincide con lo descrito en la literatura mundial. Al representar tan sólo el 3\% de todos los tumores odontogénicos, se debe enfatizar en el adecuado diagnóstico tanto clínico como radiográfico del FOA. En la actualidad, el estándar de oro es la tomografía computarizada de haz cónico, otorgando visualización con detalle de la expansión y/o perforación de corticales óseas, lo que nos permitirá evaluar el grado de agresividad de la tumoración, así como la conducta quirúrgica a seguir.

Debido a la edad de nuestra paciente, se optó por realizar un manejo quirúrgico conservador, y por las características del lecho quirúrgico no fue necesario colocar materiales de injerto óseo ni osteosíntesis reconstructiva. El manejo conservador del periostio y las corticales óseas nos aseguran una regeneración ósea satisfactoria, como lo podemos corroborar en el control postquirúrgico.

\section{BIBLIOGRAFÍA}

1. Tsuruki S, Kousuke K, Ichinokawa Y, Tsuruki T. A case of ameloblastic fibro-odontoma occupying the left maxillosinus which was enucleated by the Le Fort I osteotomy. Journal of Oral and Maxillofacial Surgery, Medicine, and Pathology. 2015; 27 (3): 380384.

2. Castellón ML, Fariña R, Martínez B, Canto L, Gunckel R. Fibroodontoma ameloblástico de la mandíbula. Rev Esp Cirug Oral y Maxilofac. 2013; 35 (2): 87-92.

3. Brierley DJ, Speight PM, Jordan RC. Current concepts of odontogenic tumours-an update. Diagnostic Histopathology. 2017; 23 (6): 266-274.

4. Ülgür II, Caduff R, Erb J, Van Waes H, Jacobsen C, Bredell MG. Ameloblastic fibro-odontoma located in the maxilla of a 3-year-old girl. Pediatr Dent J. 2014; 24 (2): 106-110.

5. Augello M, Rabufetti A, Ghazal G, Yurtsever H, Leiggener C. Ameloblastic fibro-odontoma in children. Clinical aspects and review of the literature. Oral and Maxillofacial Surgery Cases. 2017; 3 (2): 34-41.

6. Tsuji K, Yoshida H, Watanabe S, Yamamoto E, Yamada K, Tominaga K, Morita S. A case of ameloblastic fibro-odontoma accompanied with a number of impacted teeth. J Oral Maxillofac Surg Med Pathol. 2015; 27 (3): 366-368.

7. Sardana D, Rattan V, Gauba K, Goyal A, Singh S, Vai-Phei K. Surgical excision of large ameloblastic fibro-odontoma followed by alloplastic bone graft-2 year follow-up: a case report. Journal of Oral and Maxillofacial Surgery, Medicine, and Pathology. 2015; 27 (1): 41-44.

8. De Riu G, Meloni SM, Contini M, Tullio A. Ameloblastic fibro-odontoma. Case report and review of the literature. $J$ Craniomaxillofacial Surg. 2010; 38 (2): 141-144. 